\title{
Oral Psoriasis: An Overlooked Enigma
}

\author{
Mahnaz Fatahzadeh $^{\mathrm{a}}$ Robert A. Schwartz ${ }^{\mathrm{b}}$ \\ ${ }^{a}$ Department of Diagnostic Sciences, Rutgers School of Dental Medicine, and ${ }^{\mathrm{b}}$ Department of Dermatology, \\ Rutgers New Jersey Medical School, Newark, N.J., USA
}

\section{Key Words}

Oral lesions · Psoriasis · Oral manifestations · Periodontal disease

\begin{abstract}
Although cutaneous psoriasis is common, the existence of its manifestations in the oral cavity has been questioned. The definitive diagnosis of oral psoriasis can be challenging due to the variability of presentations, and overlapping clinical and histological features with a number of other conditions as well as the lack of consensus. We review oral psoriasis, noting its variable clinical appearance, delineate the differential diagnosis, and discuss management strategies.
\end{abstract}

(c) 2016 S. Karger AG, Basel

\section{Introduction}

Oral involvement by psoriasis is unusual. Kaposi wrote in 1895: 'Like Hebra, I have never seen disease analogous to psoriasis on the mucous membrane of the buccal cavity, though I have observed in some psoriatic patients gray patches which, however, were due to syphilis or corresponded to leukoplakia buccalis non-syphilitica (Schwimmer)' $[1,2]$. Although the existence of oral pso- riasis is subject to controversy [3-5], oral and other mucous membrane involvement does occur, albeit infrequently $[3,6,7]$, and particularly in association with specific subtypes of psoriasis such as generalized pustular or erythrodermic variants $[3,4]$. It is now well accepted that some patients with psoriasis manifest oral lesions synchronous with their skin disease [4], and that authentic oral manifestations share similar histopathological features with their cutaneous counterpart, plus follow a clinical course parallel with the cutaneous disease [3-5, 8-10]. A pertinent family history and positive HLA typing for either B13, B17, B37, Cw4, or Cw6 genes, often associated with psoriasis, are also considered strongly supportive of this diagnosis $[3,8,11,12]$. Isolated reports of oral lesions with characteristic histological changes, in the absence of skin psoriasis $[3,9]$, may represent manifestations of psoriasis in patients in remission from prior cutaneous disease $[4,9]$ or those with a positive family history $[4,13$, 14]. In the absence of the aforementioned diagnostic criteria, suspect oral findings may be considered psoriasiform mucositis rather than oral psoriasis [12].

The true incidence of oral involvement in psoriasis is unknown. This uncertainty, in our opinion, stems from the fact that few psoriatic patients have their oral cavities carefully examined [15]. Even less common are mucosal biopsies for known cases of psoriasis [15]. Although to

\section{KARGER}

E-Mail karger@karger.com

www.karger.com/drm
(C) 2016 S. Karger AG, Basel

$1018-8665 / 16 / 2323-0319 \$ 39.50 / 0$
Mahnaz Fatahzadeh, DMD, MSD, Professor of Oral Medicine

Department of Diagnostic Sciences, Rutgers School of Dental Medicine 110 Bergen Street

Newark, NJ 07103 (USA)

E-Mail fatahza@sdm.rutgers.edu 
our knowledge the first histologically confirmed case of oral psoriasis was reported by Oppenheim in 1903 [16], most early cases were diagnosed clinically and not biopsied for histological confirmation [10]. Thus, poor data quality questions the accuracy of incidence rates reported in the literature [10]. The low frequency of reported oral cases may also be a reflection of an accelerated rate of epithelial turnover in cutaneous lesions, approximating that of normal oral epithelium, such that oral changes are clinically subtle and difficult to recognize $[4,17]$. This lack of recognition is probably compounded by the absence of associated symptoms or modification of the clinical and histological features within the oral milieu [4]. The oral mucosa differs morphologically and immunohistologically from the cutaneous epithelium [18]. The scarcity of oral psoriasis may reflect variation in the expression of surface carbohydrates between these tissues. For example, the glycoprotein corneodesmosin, which is thought to have a role in the development of psoriasis, is found in the cutaneous but not in the mucosal epithelia $[18,19]$.

\section{Perioral and Oral Manifestations of Psoriasis}

Psoriatic involvement of the vermilion border [20,21] and perioral region is rare [22] and may occur with or without oral cavity involvement $[3,20,21,23]$. Occurrence of lip psoriasis independent of [22], synchronous with [21], or preceding the appearance of typical cutaneous lesions has been previously documented $[3,20]$. The lip vermilion is partially keratinized. Thus, psoriasis affecting the lips behaves similarly to cutaneous lesions [18]. It may present with diffuse erythema, fissuring, silvery scales and desquamation which start from the commissures and spread to involve both lips [20-22]. Occasional bleeding, serous exudate, as well as itching and discomfort aggravated by mastication and lip movements, may also be present [22]. Lip psoriasis may follow cheilitis or minimal trauma in patients with the genetic predisposition. Brenner et al. [24] reported on the induction of lip psoriasis by koebnerization in a patient whose psoriatic condition was triggered by chronic trauma from protruding maxillary dentition. Despite chronicity, the overlapping signs and symptoms, as well as the atypical site of involvement, may cause lip psoriasis to be mistaken for solar cheilosis, chronic eczema, actinic dermatitis, chronic candidiasis or leukoplakia and lead to delayed diagnosis [22].

There is no consensus regarding the authentic oral manifestations of psoriasis; however, a number of mor- phological patterns have been described [7]. These include diffuse, intense mucosal erythema associated with acute psoriatic flares, well-defined, annular, white or grayish-yellow lesions, as well as mixed, ulcerative, vesicular, pustular and indurated entities $[3,4,9,18]$. Manifestations of psoriasis may involve the oral cavity in a variety of locations $[3,9]$ with the buccal mucosa most commonly affected [4]. The palate and gingiva are unusual sites $[4,9]$. Oral findings are frequently transient, migratory, and fluctuating daily in prominence parallel to exacerbation or remission of cutaneous lesions $[4,10]$. Pinpoint bleeding reminiscent of the cutaneous Auspitz's sign may also be evident in the affected mucosa [4, 14].

A higher prevalence of benign migratory glossitis (BMG) and fissured tongue (FT) in psoriatic patients compared to the general population has also been cited in many studies $[5,8,25,26]$, prompting some clinicians to consider these oral findings as 'oral psoriasis' $[4,8,11]$. Geographic tongue, BMG or wandering rash of the tongue is a common inflammatory condition of unknown etiology affecting the dorsum and lateral borders of the tongue [27]. It develops from localized desquamation of filiform papillae resulting in multifocal erythematous patches surrounded by white, raised serpentine borders which expand centrifugally and appear to change in shape, size, location, and color over time $[8,15,27]$. Affected patients are often unaware since lesions are typically asymptomatic [27]. Periodic exacerbations characterized by burning, particularly upon exposure to spicy foods, are possible. An extraglossal counterpart of BMG affecting nonkeratinized oral mucosal surfaces is referred to as ectopic geographic tongue, migratory stomatitis and erythema circinata migrans $[8,10,15]$.

The identical histopathological features between BMG, erythema circinata migrans and cutaneous pustular psoriasis provide support for the notion that these oral changes represent oral psoriasis $[4,10,18]$. The immunohistochemical studies also illustrate that the makeup of the subepithelial infiltrate in BMG resembles that of psoriatic skin lesions (i.e. predominance of $\mathrm{CD} 4$-positive cells in the macrophage and T-cell infiltrate) [28]. Moreover, the resolution of both BMG and cutaneous lesions with antipsoriatic agents appears to suggest a common etiology [29]. Despite these observations, recognition of geographic tongue as a true oral expression of psoriasis has been questioned, particularly when the existence of geographic tongue predates cutaneous psoriasis by many years.

FT, also known as lingua plicata or scrotal tongue, is a condition characterized by the presence of anteroposteri- 
orly oriented groove(s) with lateral extensions on the dorsal tongue. Ulmansky et al. [11] postulated that BMG represents a transient and FT a delayed, more stable expression of oral psoriasis. The increased incidence of FT with age lends credence to the proposed evolution of FT from BMG $[5,11]$. However, the common and independent occurrences of psoriasis, FT and BMG in the population drive some to consider their concomitant presence as coincidental $[5,7]$. FT and BMG may also represent nonpathognomonic oral changes which are more likely to develop in the context of cutaneous psoriasis [7]. Opponents posit that FT, the most commonly reported oral finding amongst psoriatic patients [15], does not have the characteristic histopathology [15] and attribute the observed association between BMG, FT and generalized pustular psoriasis to sharing of common genes and a polygenetic mode of inheritance [5]. Interestingly, both psoriasis and BMG appear to be associated with HLACw6 [15].

\section{Differential Diagnosis}

The clinical differential diagnosis for oral psoriasis is extensive and includes a number of inflammatory, ulcerative, blistering and infectious conditions $[4,9,18]$. In particular, clinical distinction between candidiasis, Reiter's syndrome, inflammatory conditions such as BMG, erythema circinata and oral psoriasis - collectively referred to as psoriasiform disorders - can be challenging $[4,7,10,18]$. In addition, mucosal abnormalities caused by ill-fitting prostheses, cheek biting or chronic smoking may resemble oral psoriasis [4]. In fact, such sources of oral irritation could promote an initiation of new lesions by the Köbner phenomenon $[4,8,9]$.

The clinical presentation of oral candidiasis may overlap with that of oral psoriasis. For example, focal or generalized psoriatic erythematous patches of the oral mucosa clinically resemble atrophic candidiasis or denture-associated stomatitis $[3,14]$. In addition, hyperplastic rete ridges and intraepithelial neutrophils are shared histopathological features for both psoriasis and candidiasis. Manifestation of oral psoriasis as diffuse erythema with granular texture confined to the denture-bearing mucosa has been reported $[3,14]$. When indicated, a negative PAS stain of a superficial smear or tissue section from the affected mucosa or failure of response to topical and systemic antifungals may help exclude a fungal etiology $[3,14]$.

Reiter's syndrome, the most common type of inflammatory polyarthritis, is a genetic condition expressed in response to gastrointestinal or genitourinary infections [4]. This reactive form of arthritis is characterized by urethritis, conjunctivitis and arthritis in genetically predisposed individuals (HLA-B27 positivity) $[3,4]$. Overlap of cutaneous, rheumatological, histological, and radiographic features renders differentiation between psoriasis and reactive arthritis difficult [6]. In fact, many experts consider that Reiter's syndrome is best classified as a type of psoriasis [6]. Classic cutaneous lesions of Reiter's syndrome, known as keratoderma blenorrhagicum, clinically resemble pustular psoriasis [6], and when affecting the palms and soles, are essentially the same as palmoplantar pustular psoriasis [4]. The mucosal lesions of psoriasis and reactive arthritis are also difficult to differentiate. These include circinate balanitis and ulcerative vulvitis, which affect the genital mucosa, as well as erythematous plaques, palatal erosions, ulcerations, glossitis and geographic tongue, which affect mucous membranes in the oral cavity. These are often painless and frequently overlooked [6]. Other similarities between the two conditions include arthritic manifestations, negative serology for rheumatoid factor and antinuclear antibody as well as the potential for a flare-up related to an antecedent infection $[6,12]$.

\section{Histopathological Features of Oral Psoriasis}

Although a tissue biopsy is necessary as part of the workup process, there is no consensus regarding the microscopic features of oral psoriasis [7]. In addition, microscopic examination is insufficient for the differentiation between psoriasiform disorders. This is because their histopathological features, known as psoriasiform mucositis (fig. 1), closely resemble those of cutaneous psoriasis [7, $18]$, albeit being less florid $[4,10,30]$. Nevertheless, a tissue biopsy for histopathology and immune studies are useful to exclude vesiculobullous conditions [18]. Characteristic histopathological features of dermal psoriasis include epithelial acanthosis, elongation and clubbing of rete ridges, dilation of superficial capillaries, thinning of suprapapillary epithelium and intraepithelial inflammatory infiltrate with or without Munro's abscesses $[3,4,18]$. The latter are clusters of neutrophils in the upper layers of the epithelium and are frequently absent in older lesions an observation emphasizing that age and activity of lesions may impact their microscopic appearance $[4,10,18]$.

It is notable that the histopathological criteria used for the diagnosis of cutaneous psoriasis may not be fully applicable to its oral counterpart [7]. For example, Munro's abscesses, although neither essential nor specific to the di- 

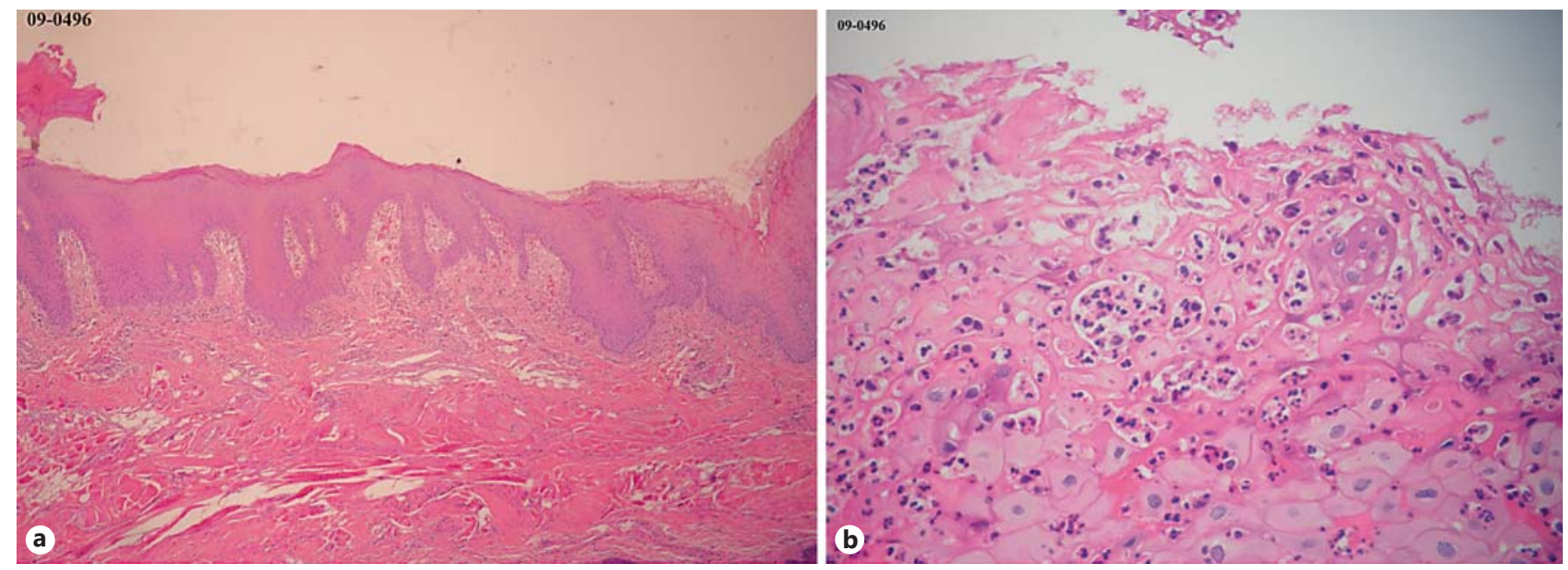

Fig. 1. Psoriasiform mucositis. a Histological photomicrograph illustrating hyperkeratotic squamous epithelium with elongated rete ridges, dilated blood vessels in the papillary area, and intraepithelial inflammatory cells. HE. $\times 100$. b Prominent neutrophilic infiltration and microabscesses (Munro's abscesses) in the superficial epithelium. HE. $\times 400$.

agnosis [4], may be difficult to be discerned in the psoriatic lesions of movable oral mucosa where the stratum corneum is absent, thus possibly hindering a diagnosis [4, 30]. Other pustular conditions of oral mucosa include parulis related to an abscess of odontogenic origin, subcorneal pustular mucositis, pyostomatitis vegetans, and herpetiform stomatitis [27]. Neutrophilic infiltration or microabscesses in the latter two conditions involve the lower layers of the epithelium or subjacent connective tissue [27]. In subcorneal pustular mucositis, there is separation of keratin from the spinous layer, which is helpful in its differentiation from psoriasiform mucositis [27]. Considering the preceding discussion, diagnosis of oral psoriasis relies on clinicopathological correlation $[4,10,30]$.

Other oral findings described in association with psoriasis include angular cheilitis, enlarged fungiform papilla, gingivitis, and periodontitis $[4,11,31,32]$. Yamada et al. [33] reported focal changes in the gingival mucosa and breakdown of proximal periodontal tissues associated with the flares of cutaneous disease, suggesting a potential role for gingival psoriasis in the pathogenesis of the periodontal disease. In a recent study, the majority of the 60 psoriatic patients had gingivitis compared to only 10 of the 45 healthy subjects examined by a trained oral pathologist [31]. Also, 23 of the patients with psoriasis had periodontal disease compared to 9 of 45 healthy subjects [31]. In addition, salivary expression of proinflammatory cytokines such as TNF- $\alpha$, TGF- $\beta_{1}$, MCP- 1 and IL- $1 \beta$ was significantly higher in the psoriatic patients compared to the control group, and the severity of oral disease correlated well with the expression of TGF- $\beta_{1}$, IL- $1 \beta$ and MCP1 in saliva [31]. These observations suggest that a common inflammatory process may account for both psoriasis and periodontal disease. Therefore, examination of the oral cavity in psoriatic patients could not only help clinicians better appreciate the true incidence of oral involvement, but also identify and treat any associated oral disease [31]. Assessment of specific inflammatory markers may also be relevant for evaluating the extent of oral disease in the affected patients [31]. Both secretion and concentration of salivary IgA and lysozyme, biomarkers critical to mucosal immunity, are lower in psoriatic patients compared to controls $[32,34]$. This finding may not only predispose patients with psoriasis to microbial infection, but also portends an increased risk of precipitating the disease itself [34]. One may also speculate that controlling the biofilms responsible for the initiation and perpetuation of periodontal disease may, in turn, help reduce the likelihood of triggering psoriasis [32].

\section{Treatment}

Management of oral psoriasis is not well described primarily because, unlike cutaneous psoriasis, most cases of oral psoriasis are asymptomatic, transient and not requir- 
Table 1. Proposed algorithm for evaluation and management of oral psoriasis

Evaluation

Regularly screen the oral cavity of psoriatic patients for:

Mucosal alterations suspicious for oral psoriasis [4]

Adequacy of oral hygiene $[4,8,9,17]$

Presence of oral irritants which may promote new lesions $[4,8,9,17]$

Ill-fitting prostheses

Orthodontic appliances

Sharp, broken teeth

Missing dental restorations

Oral parafunction

Presence of oral diseases [31]

Potential adverse effects of antipsoriatic medications $[8,17]$

Possible appropriate referral for oral health assessment and management

Biopsy symptomatic oral mucosal alterations for microscopic evaluation in patients with current or a history of psoriasis. Diagnosis relies on $[3,4,8,9,11,12,14]$ :

Detailed history regarding preexisting and/or current cutaneous psoriasis

Family history of the disease

Clinical and histological evidence

Exclusion of other potential causes of oral findings

HLA typing, as indicated

\section{Education}

Reassure patients about the benign nature of the condition, especially when malignancy is a concern [30]

Inform patients about the chronic nature of psoriasis and the potential for recurrent oral changes [11]

Counsel patients to avoid acidic, spicy foods, particularly in the presence of oral dryness and stomatodynia [8]

Encourage smoking cessation [8]

Emphasize thorough yet gentle oral hygiene $[4,9,13,14]$

Recommend modified oral hygiene aids when psoriatic arthritis hinders adequate oral hygiene [17]

Demonstrate how to self-examine the oral cavity for mucosal changes between visits

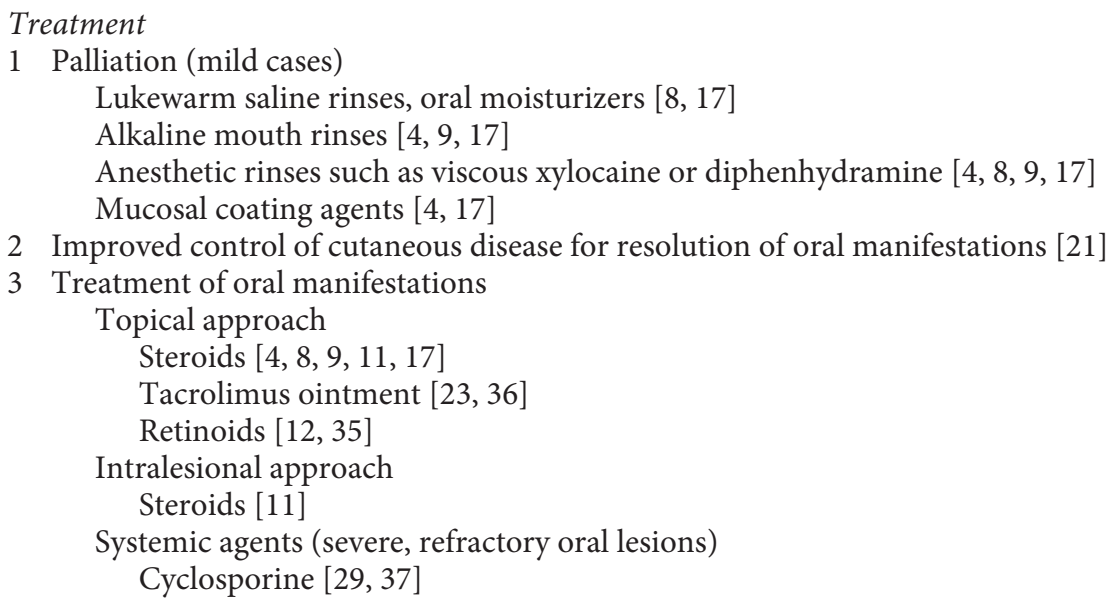

ing specific interventions [4]. Also, control of cutaneous disease often helps resolve symptomatic oral psoriasis, which may occur during a flare. Typical symptoms, when present, may include oral pain, burning or changes in taste perception [13, 17, 35]. Patients may also express concern about the malignant potential of lesions or the unaesthetic appearance of the affected visible mucosa $[30,35]$.
Although many of the therapeutic modalities for cutaneous psoriasis are not appropriate for use in the oral cavity [11], a variety of approaches have been documented. Administration of topical or intralesional steroids guided by the severity of symptoms and localization of lesions are generally efficacious $[3,9,11]$. It is important to note that oral psoriasis may not only resemble oral candidiasis, but the latter could also represent a concomitant superinfec- 
tion complicating both diagnosis and management, particularly when corticosteroid therapy is a consideration $[4,9]$. Topical application of $0.1 \%$ tretinoin solution has proven effective in controlling symptomatic BMG in numerous patients [35]. Resolution of BMG and psoriatic lesions confined to the lip vermilion with topical application of $0.1 \%$ tacrolimus ointment has also been documented [22, 36]. Abe et al. [29] reported a therapeutic response to the administration of a systemic cyclosporine microemulsion preconcentrate of $3 \mathrm{mg} / \mathrm{kg} /$ day for the management of severely symptomatic, refractory BMG in a 54-year-old woman [37].

Both steroids and retinoids help control the excessive epithelial turnover responsible for disease pathogenesis [10]. Considering the nature of a subepithelial infiltrate in psoriatic lesions, the efficacy of tacrolimus is likely a reflection of the local inhibition of T-cell activation and downregulation of cytokines which disrupt epithelial hemostasis [22]. Antipsoriatic efficacy of cyclosporine may also explain its efficacy in controlling T-cell-mediated alterations of oral mucosal epithelium [36]. Severe oral manifestations of psoriasis, particularly in association with other sites of involvement, may require systemic intervention. Gul et al. [21] achieved control of psoriasis affecting the lips, nails and vulva with oral methotrexate.

Despite efficacy, a number of medications used to treat psoriasis may lead to adverse effects in the oral cavity. Examples include xerostomia associated with retinoids, gingival enlargement with cyclosporine, and stomatitis associated with methotrexate $[8,17]$. Patients with psoriatic arthritis or severe cutaneous involvement may also have difficulty holding a toothbrush or performing adequate oral hygiene [17]. Therefore, regular oral evaluations are necessary to prevent, detect and manage oral disease as well as potential adverse effects of antipsoriatic medications in the oral cavity. Table 1 provides a summary of diagnostic and management algorithms for oral psoriasis.

\section{Conclusion}

Despite the common prevalence of psoriasis, our knowledge regarding its authentic oral manifestations is limited. This situation is partially a reflection of the rarity and transient nature of the oral alterations compounded by the absence of clinical and histopathological consensus for the diagnosis of oral psoriasis. Prospective interdisciplinary studies are needed to clarify the relationship between entities suspected to be a counterpart of cutaneous psoriasis in the oral cavity. Until then, diagnostic approaches should rely on a detailed history regarding preexisting and/or current cutaneous psoriasis, family history of the disease, clinical and histological evidence, HLA typing when indicated, and the exclusion of other potential causes for oral findings. This is particularly relevant when the recent onset of oral signs and symptoms could mask a potential association between the longstanding or distant cutaneous psoriasis and its oral counterpart. In patients with known psoriasis, routine examination of the skin should include the oral mucosa with the intention to identify subtle changes which may represent oral psoriasis. Examination of the skin for psoriatic changes could also provide insight into the etiology of oral lesions suspected or diagnosed as psoriatic. Although often asymptomatic, oral psoriasis may cause oral discomfort and concern for the patient. Therefore, clinicians should be familiar with the spectrum of oral signs and symptoms, diagnostic workup, and management strategies for symptomatic oral psoriasis.

\section{Disclosure Statement}

The authors have no conflicts of interest to disclose.

\section{References}

1 Kaposi M: Pathology and Treatment of Diseases of the Skin for Practitioners and Students. Translation of the Last German Edition. New York, Wood \& Co, 1895, p 413.

2 Schuppner JJ: Clinical picture of mucosal involvement in psoriasis pustulosa. Arch Klin Exper Dermatol 1960;209:600-607.

-3 Younai SF, Phelan JA: Oral mucositis with features of psoriasis: report of a case and review of the literature. Oral Surg Oral Med Oral Pathol Endod 1997;84:61-67.
4 Bruce AJ, Rogers RS III: Oral psoriasis. Dermatol Clin 2003;21:99-104.

5 Pérez F, Aveldañez A, Ruvalcaba M, Barcelot M, Camacho M, Memije M, Taylor A: Prevalence of oral lesions in patients with psoriasis. Med Oral Pathol Oral Cir Bucal 2008;13: e703-e708.

6 Wu IB, Schwartz RA: Reiter's syndrome: the classic triad and more. J Am Acad Dermatol 2008;59:113-121.
7 Mattsson U, Warfvinge G, Jontell M: Oral psoriasis - a diagnostic dilemma: a report of two cases and a review of the literature. Oral Surg Oral Med Oral Pathol Oral Radiol 2015; 120:e183-e189.

8 Zhu J-F, Kaminski MJ, Pulitzer DR, Hu J, Thomas HF: Psoriasis: pathophysiology and oral manifestations. Oral Dis 1996;2:135-144.

9 Brice DM, Danesh-Meyer MJ: Oral lesions in patients with psoriasis: clinical presentation and management. J Periodontol 2000;71: 1896-1903. 
10 Weathers DR, Baker G, Archard HO, Jefferson Burke E: Psoriasiform lesions of the oral mucosa (with emphasis on 'ectopic geographic tongue'). Oral Pathol 1974;37:872-888.

11 Ulmansky M, Michelle R, Azaz B: Oral psoriasis: report of six new cases. J Oral Pathol Med 1995;24:42-45.

-12 Reis V, Artico G, Seo J, Bruno I, Hirota S, Lemos C, Martins M, Migliari D: Psoriasiform mucositis on the gingival and palatal mucosae treated with retinoic-acid mouthwash. Int J Dermatol 2013;52:113-125.

13 Picciani B, Silva-Junior G, Carneiro S, Sampaio A, Goldemberg D, Oliveira J, Porto L, Dias E: Geographic stomatitis: an oral manifestation of psoriasis? J Dermatol Case Rep 2012;6:113-116.

14 Wooten JW, Tarsitano JJ, Lavere AM: Oral psoriasiform lesions: a possible prosthodontic complication. J Prosthet Dent 1970;24:145147.

15 Daneshpazhooh M, Moslehi H, Akhyani M, Etesami M: Tongue lesions in psoriasis: a controlled study. BMC Dermatol 2004;4:16.

16 Oppenheim M: Psoriasis mucosae oris Monatsschr Prakt Dermatol 1903;37:481.

17 Dreyer LN, Cohen Brown G: Oral manifestations of psoriasis: clinical presentation and management. NY State Dent J 2012;78:14-18.

18 Yesudian PD, Chalmers RJ, Warren RB, Griffiths CE: In search of oral psoriasis. Arch Dermatol Res 2012;304:1-5.

19 Allen M, Ishida-Yamamoto A, McGrath J, Davison S, Lizuka H, Simon M, Guerrin M, Hayday A, Vaughan R, Serre G, Trembath R, Barker J: Corneodesmosin expression in psoriasis vulgaris differs from normal skin and other inflammatory skin disorders. Lab Invest 2001;81:969-976
20 Baz K, Yazici AC, Usta A, Ikizoglu G, Apa DD: Isolated lip involvement in psoriasis. Clin Exp Dermatol 2007;32:578-579.

21 Gul U, Kilic A, Gonul M, Soylu S, Bilgili S, Han O: Psoriasis of the lips: an unusual localization. Int J Dermatol 2006;45:1381-1382.

22 Rahman MA, Fikree M: Perioral psoriasis. J Eur Acad Dermatol Venereol 2000;14:521522.

23 Yamamoto T, Nishioka K: Successful treatment with topical tacrolimus for oral psoriasis. J Eur Acad Dermatol Venereol 2006;20: 1133-1167.

24 Brenner S, Lipitz R, Ilie B, Krakowski A: Psoriasis of the lips: the unusual Köbner phenomenon caused by protruding upper teeth. Dermatologica 1982;164:413-416.

25 Costa SC, Hirota SK, Takahashi MD, Andrade H Jr, Migliari DA: Oral lesions in 166 patients with cutaneous psoriasis: a controlled study. Med Oral Patol Oral Cir Bucal 2009; 14:371-375.

26 Arvind Babu RS, Chandrashekar P, Kumar KK, Sridhar G, Lalith K, Rao V, Reddy B: A study on oral mucosal lesions in 3,500 patients with dermatological diseases in South India. Ann Med Health Sci Res 2014;4:84-93.

27 Tarakji B, Umair A, Babaker Z, Sn A, Gazal G, Sarraj F: Relation between psoriasis and geographic tongue. Clin Diagn Res 2014;8: 11:ZE06-ZE07.

28 Baker BS, Swain AF, Valdimarsson H, Fry L: T-cell subpopulations in the blood and skin of patients with psoriasis. Br J Dermatol 1984; 110:37-44
29 Abe M, Sogabe Y, Syuto T, Ishibuchi H, Yokoyama Y, Ishikawa O: Successful treatment with cyclosporin administration for persistent benign migratory glossitis. J Dermatol 2007;34:340-343.

30 Ariyawardana A, Tilakaratne W, Ranasinghe A, Dissanayake M: Oral psoriasis in an 11-year-old child: a case report. Int J Pediatr Dent 2004;14:141-145.

31 Ganzetti G, Campanati A, Santarelli A, Pozzi V, Molinelli E, Minnetti I, Brisigotti V, Procaccini M, Emanuelli M, Offidani A: Involvement of the oral cavity in psoriasis: results of a clinical study. Br J Dermatol 2015; 172:282-285

32 Ganzetti G, Campanati A, Santarelli A, Pozzi V, Molinelli E, Minnetti I, Brisigotti V, Procaccini M, Emanuelli M, Offidani A: Periodontal disease: an oral manifestation of psoriasis or an occasional finding? Drug Dev Res 2014;75(suppl 1):S46-S49.

33 Yamada J, Amar S, Petrungaro P: Psoriasisassociated periodontitis: a case report. J Periodontal 1992;63:854-857.

34 Koh D, Yang Y, Khoo L, Nyunt SZ, Ng V, Goh CL: Salivary immunoglobulin A and lysozyme in patients with psoriasis. Ann Acad Med Singapore 2004;33:307-310.

35 Helfman RJ: The treatment of geographic tongue with topical Retin-A solution. Cutis 1979;24:179-180.

36 Ishibashi M, Tojo G, Watanabe M, Tamabuchi T, Masu T, Aiba S: Geographic tongue treated with topical tacrolimus. J Dermatol Case Rep 2010;4:57-59.

37 Nakagawa H, Aiba S, Asahina A, et al: A consensus conference report on psoriasis therapy with cyclosporine MEPC. Jpn J Dermatol 2004;114:1093-1105. 